\title{
The cell-bound hemolysin of Serratia marcescens contributes to uropathogenicity
}

\author{
R. Marre, 'J. Hacker ${ }^{2}$ and V. Braun ${ }^{3}$ \\ 'Institut für Medizinische Mikrobiologie der Medizinischen Universität zu Lübeck, F.R.G.; \\ ${ }^{2}$ Institut für Genetik und Mikrobiologie der Universität Würzburg. F.R.G., and ${ }^{3}$ Lehrstuhl für \\ Mikrobiologie // der Universität Tübingen, F.R.G.
}

(Received June 30, 1989; accepted July 3, 1989)

\begin{abstract}
Marre, R. (Institut für Medizinische Mikrobiologie der Medizinischen Universität zu Lübeck, F.R.G.), J. Hacker and V. Braun. The cell-bound hemolysin of Serratia marcescens contributes to uropathogenicity. Microbial Pathogenesis 1989; 7: 153-156.

The contribution of the cell-bound hemolysin of Serratia marcescens to uropathogenicity was studied in an experimental urinary tract infection in rats. The strain carrying the Serratia hemolysin colonized the urinary tract more and lead to a stronger inflammatory response compared to the isogenic hemolysin negative strain.
\end{abstract}

Key words: Serratia marcescens; uropathogenicity; hemolysin; rat.

\section{Introduction}

Serratia marcescens is an important pathogen causing predominantly nosocomial infections in immunocompromised patients ranging from urinary tract infections to endocarditis, meningitis and bacteremia. Pathogenicity of this microorganism has been associated with a formation of fimbriae, ${ }^{1,2}$ the production of proteases, which degrade various serum proteins, ${ }^{1,3,4}$ the presence of different cell wall antigens ${ }^{5}$ and the ability to resist the bactericidal action of serum. ${ }^{6}$ Recently Braun et al. ${ }^{7}$ noticed that Serratia marcescens strains rapidly lyse human erythrocytes in solution. On blood agar Serratia colonies show a narrow zone of a faint hemolysis. The Serratia hemolysin has been shown to differ markedly from the well characterized $E$. coli hemolysin. ${ }^{8}$ It is predominantly cell associated, requires actively metabolising bacteria and does not need calcium ions for activity. The hemolysin determinant is contained on a $7.5 \mathrm{~kb}$ fragment of chromosomal DNA and shows two open reading frames designated shlA which encodes the hemolysin protein and $s h / B$ which somehow activates shIA. ${ }^{9}$ Since there has been no study on the role of the Serratia hemolysin in experimental infections, we investigated the contribution of this hemolysin to uropathogenicity in an experimental rat model. ${ }^{10}$ 


\section{Results and discussion}

As demonstrated in Fig. 1 renal colonization of the strain producing the Serratia hemolysin was more than five times higher than that of the Shl-negative recipient strain $(P<0.05)$. Bladder colonizaton was distinctly higher than renal colonization in both groups of rats with a tendency to higher colony counts in the group infected with 536/21 pES2. The mean bladder weights of animals infected with 536/21 pES2 were more than double as high as in the control group $(0.30$ vs $0.14 \mathrm{~g})$. Numbers of leukocytes in the urine were 2.6 times higher in the animals colonized with $E$. coli $536 / 21 \mathrm{pES} 2$.

It is obvious from the data presented here that the Serratia hemolysin contributes to uropathogenicity in an experimental rat pyelonephritis model. Shl-positive bacteria lead to higher renal colonization than their non-hemolytic counterparts. Bladder colonization was also affected by the Serratia hemolysin but to a lesser extent. The contribution of Shl to inflammatory responses was indicated by an increase of leukocyturia and thickening of the bladder walls. These results are in line with those of König et al. ${ }^{11}$ who were able to demonstrate that $\mathrm{Shl}$ induces the release of leukotriene from polymorphonuclear leukocytes, the release of histamine from rat mast cells, and chemiluminescence of neutrophils.

The extent of contribution of Shl to uropathogenicity was similar to that of the secreted $E$. coli alpha hemolysin derived from an $E$. coli 018 strain. ${ }^{10}$ It shows that a hemolysin which is predominantly cell bound and rapidly inactivated in non-growing bacteria, also exerts biological effects. The hemolysin might facilitate the destruction of the epithelium of the urinary tract and the tubuli and thus facilitate invasion of urinary tract tissue.

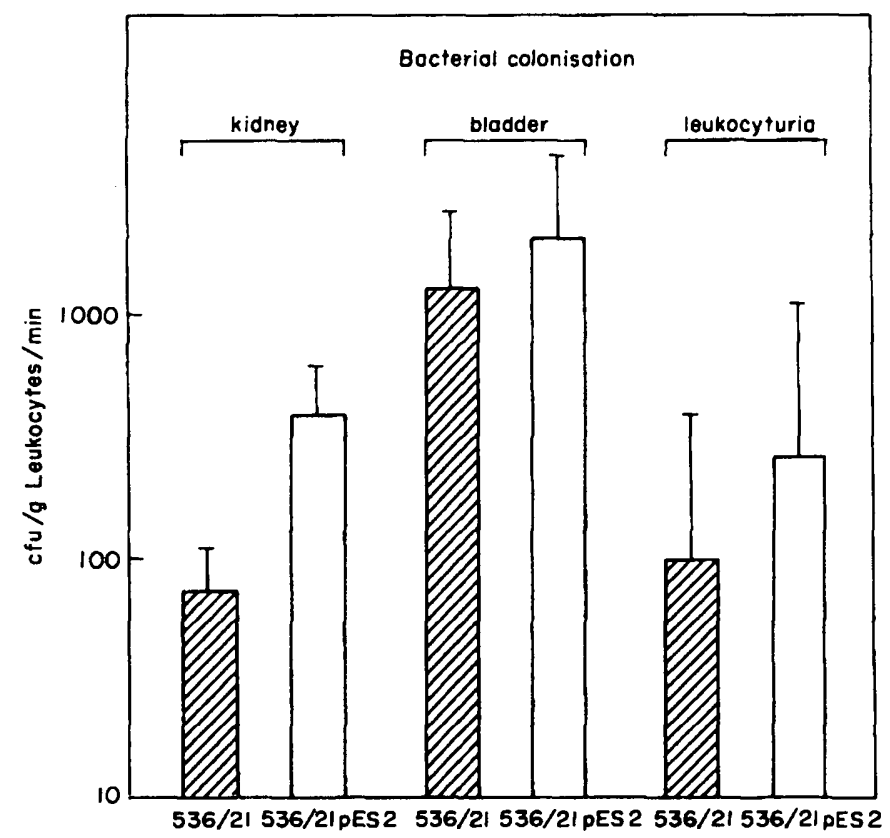

Fig. 1. Bacterial colonization of kidney and bladder by $E$. coli strain $536 / 21$ (number of rats 36 ) and the transformant 536/21 pES2 (number of rats 40), which carried the determinant of the Serratia hemolysin. Each column gives the mean value of cfu/g of tissue. Bars indicate the standard error. Leukocyturia (number of rats per strain $=5$ ) was calculated as number of cells eliminated per min (median+standard error). Each column represents the median of leukocytes eliminated per $\mathrm{min}$, the bars indicate the standard error. 
In this study the role of Serratia hemolysin in infection was demonstrated after transformation of a Shl-coding plasmid into an $E$. coli carrier strain which is well adapted to the animal system used. Studies are underway to demonstrate whether or not the Serratia hemolysin also plays a role in the virulence of its natural host bacteria.

\section{Materials and methods}

Strains. Since it was at first easier to construct isogenic pairs of $E$. coli than of Serratia which only differed in hemolytic activity, the well-characterized $E$. coli strain 536/21 $06 \mathrm{~K} 15$ was used as a carrier strain for the recombinant Serratia hemolysin. This strain was previously used in this model of infection for studies on uropathogenicity and was able to colonize the rat urinary tract. The strain has been described in detail. ${ }^{10}$ Strain $536 / 21$ was transformed with plasmid pES2, which carried the cloned chromosomal hemolysin determinant of Serratia marcescens. The transformant showed a narrow zone of faint hemolysis on sheep blood agar similar to that of Seratia marcescens. With respect to other possible virulence factors such as serum resistance, fimbriae, cell wall antigens and growth rates strain 536/21 and transformant 536/21 pES2 were identical.

Methods. For initiation of a urinary tract infection $1.5 \mathrm{ml}$ of a suspension of the bacteria to be tested (concentration $10^{7} \mathrm{cfu} / \mathrm{ml}$ ) was injected via urethra into the bladder of the rats (strain: Han WIST).$^{10}$ This resulted in a vesicoureteral reflux and subsequent colonization of the kidneys. Depending on the virulence of the strains used, a persisting pyelonephritis might develop. One week after the infection the rats were sacrificed, kidneys and bladder removed aseptically, the bladders were opened and rinsed in sterile saline. Both organs were homogenized, appropriately diluted and viable counts were made on McConkey agar. The identity of the isolated colonies with the $E$. coli strains injected was tested serologically and by the presence of the chromosomally encoded streptomycin resistance. The majority of the reisolated bacteria still contained the plasmid with the hemolysin gene. The mean number of $\mathrm{cfu} / \mathrm{g}$ of tissue of each group was calculated and served as a measure of uropathogenicity. Forty rats were used in each group. In order to evaluate leukocyturia, five rats of each group received $10 \mathrm{ml}$ of $0.9 \%$ saline and furosemide $(10 \mathrm{mg} / \mathrm{kg})$ intraperitoneally on the sixth day of infection. The rats were placed singly into metabolic cages for $2 \mathrm{~h}$. The urine was collected and the number of leukocytes determined. The cell elimination per min was calculated. The statistical evaluation was performed according to Kruskal-Wallis followed by the Nemenyi-test, if statistically significant differences $(P<0.05)$ were found. ${ }^{12}$

This work was supported by the Deutsche Forschungsgemeinschaft.

\section{References}

1. Kohno K, Yamamoto T, Kuroiwa A, Amako K. Purification and characterization of Serratia marcescens US5 pili. Infect Immun 1984; 46: 295-300.

2. Clegg S, Hull S, Hull R. Pruckler J. Construction and comparison of recombinant plasmids encoding type 1 fimbriae of members of the family Enterobacteriaceae. Infect Immun 1985; 48: 275-9.

3. Matsumoto K, Maeda H, Takata K, Kamata R, Okamura R. Purification and characterization of four proteases from a clinical isolate of Serratia marcescens kums 3958. J Bacteriol 1984; 157: 225-32.

4. Molla A, Matsumoto K, Oyamada I, Katsuki T, Maeda H. Degradation of protease inhibitors immunoglobulin and other serum proteins by Serratia protease and its toxicity to fibroblasts in culture. Infect Immun 1986; 53: 522-9.

5. Traub WH. Virulence of nosocomial isolates of Serratia marcescens for NMRI mice: correlation with O-antigens 06 and 014. Zentralbl Bakteriol Mikrobiol Hyg [B] 1982; 252: 360-9.

6. Gutschik E, Norwood RS, Moller S. Olling S. Experimental endocarditis in rabbits. 4. Experiments with Serratia marcescens on the significance of serum susceptibility and proteolytic capacity of the strains and the influence of an indwelling catheter. Acta Pathol Microbiol Immunol Scand [B] 1980; 88: 269276.

7. Braun V, Günther H, Neuß B, Tautz C. Hemolytic activity of Serratia marcescens. Arch Microbiol 1985; 141: $371-6$.

8. Braun V, Neuß B, Huan Y, Schiebel E, Schöffler H, Jander G. Identification of the Serratia marcescens hemolysin determinant by cloning into Escherichia coli. J Bacteriol 1987; 169: 2113-20. 
9. Poole K, Schiebel E, Braun V. Molecular characterization of the hemolysin determinant of Serratia marcescens. J Bacteriol 1988; 170: 3177-88.

10. Marre R, Hacker J, Henkel W, Goebel W. Contribution of cloned virulence factors from uropathogenic Escherichia coli strains to nephropathogenicity in an experimental rat pyelonephritis model. Infect Immun 1986; 54: 761-67.

11. König W, Faltin $Y$, Scheffer J, Schöffler H, Braun V. Role of cell-bound hemolysin as a pathogenicity factor for Serratia infections. Infect Immun 1987; 55: 2554-61.

12. Diehl JM, Kohr HU. Durchführungsanleitungen für statistische Tests. Verlag Chemie Weinheim, 1977. 